\title{
Frontline Perspectives on Physician Burnout and Strategies to Improve Well-Being: Interviews with Physicians and Health System Leaders
}

\author{
Ellis C. Dillon, $\mathrm{PhD}^{1,2}$, Ming Tai-Seale, $\mathrm{PhD}, \mathrm{MPH}^{3}, \mathrm{Amy}$ Meehan, $\mathrm{MPH}^{1,2}$, \\ Veronique Martin, $P h D, M P H^{1,2}$, Robert Nordgren, MD, MBA, MPH ${ }^{4}$, Tim Lee, $M D^{4}$, \\ Teresa Nauenberg, $M D^{4}$, and Dominick L. Frosch, $P h D^{1,2}$ \\ ${ }^{1}$ Center for Health Systems Research, Sutter Health, Palo Alto, CA, USA; ${ }^{2}$ Palo Alto Medical Foundation Research Institute, Palo Alto, CA, USA; \\ ${ }^{3}$ University of California San Diego, San Diego, CA, USA; ${ }^{4}$ Palo Alto Foundation Medical Group, Palo Alto, CA, USA.
}

BACKGROUND: Nationally over 50\% of physicians report symptoms of burnout.

OBJECTIVE: To understand the perspectives of health system leaders and frontline physicians on contributors to physician burnout and strategies to improve wellbeing.

DESIGN: We conducted in-depth interviews with health system leaders and frontline physicians at a large, predominantly fee-for-service, multispecialty group practice with approximately 1300 physicians.

PARTICIPANTS: The 17 participants included 15 physicians, (12 Internal Medicine and Family Medicine physicians and 3 from other specialties), 11 individuals in leadership roles, and 11 women.

APPROACH: Interviews included a review of factors associated with burnout at the organization, asking participants which factors they believed contributed to burnout, questions about experiences of burnout, and what specific changes would improve well-being.

KEY RESULTS: All 17 participants agreed that organizational factors were key contributors to burnout, while only 9 mentioned the salience of individual factors: "It does not matter how resilient or positive you are, the work environment, especially in primary care will eventually be a problem." An increasing workload associated with the electronic health record (EHR) and a culture focused on productivity were cited as contributing to burnout, especially among physicians in Internal Medicine and Family Medicine (primary care) departments. Physicians in primary care, women, and leaders described multiple barriers to well-being. Participants described responding to increased workloads by reducing clinical work hours. Participants suggested reducing and compensating EHR work, expanding care teams/support staff, reducing use of metrics, providing more support to leaders, changing the business model, and increasing positivity and collegiality, as essential to improving well-being.

CONCLUSION: Interviews reveal a variety of interacting factors contributing to physician burnout. Reducing

Electronic supplementary material The online version of this article (https://doi.org/10.1007/s11606-019-05381-0) contains supplementary material which is available to authorized users.

Received February 12, 2019

Accepted August 30, 2019

Published online October 28, 2019 clinical work hours has become a coping strategy. Changes recommended to improve physician well-being include increasing support staff, reducing EHR workload, changing revenue generation and compensation approaches, and shifting organizational culture to place more value on physician wellness.

KEY WORDS: physician; burnout; well-being; interviews; qualitative.

J Gen Intern Med 35(1):261-7

DOI: $10.1007 / \mathrm{s} 11606-019-05381-0$

(c) Society of General Internal Medicine 2019

\section{INTRODUCTION}

In recent decades, the physician's role has changed owing to the organizational transformation of health care, the growth of health systems, misaligned financial incentives, and the adoption of electronic health records (EHR). ${ }^{1-3}$ These changes have contributed to an escalating crisis of physician burnout. ${ }^{4}$ In $2014,54 \%$ of physicians in a nationally representative sample reported symptoms of burnout, an increase from $46 \%$ in 2011 , and double the rate found in the general United States population. ${ }^{4}$ Physicians in primary care are at increased risk of burnout. ${ }^{4-7}$

Burnout affects personal relationships and increases the risk of alcoholism and suicide. ${ }^{8,9}$ It also poses problems for health systems and patients via increased professional turnover, decreased productivity, reduced quality and safety of patient care, ${ }^{10-14}$ and lowered patient satisfaction. ${ }^{15,16}$ In order to achieve the triple aim of improving patient experience and population health whilst reducing the cost of health care, some have argued the need to include physician well-being, making it the quadruple aim. ${ }^{17,18}$ Health systems are increasingly taking steps to enhance physician well-being. ${ }^{19,20}$ For instance, the Mayo Clinic has used a bottom-up approach to tackling burnout using focus groups with physicians. ${ }^{21}$ Other approaches target individual factors such as promoting resilience and mindfulness. ${ }^{22,23}$

Most qualitative studies on physician burnout investigate the individual factors influencing burnout. ${ }^{24-26}$ Other studies analyze survey data, which lack nuanced information about the experience of burnout and do not typically explore strategies to improve well-being. A recent survey of 934 physicians 
in our organization identified a number of risk factors for burnout, ${ }^{27}$ and this research is a qualitative companion study aimed at understanding perspectives of frontline physicians and health system leaders on contributors to burnout and potential solutions.

\section{METHODS}

We conducted in-depth key informant interviews with frontline physicians and health system leaders, e.g., leaders of individual departments and specialties or executives at Palo Alto Medical Foundation (PAMF), a predominately fee-for-service, multispecialty group practice in Northern California with over 1300 physicians and serving about 1 million patients. Interview participants provided informed consent and the protocol was approved by the organization's Institutional Review Board.

Key informants were identified through organizational stakeholders and snowball sampling. Interviews were conducted by three researchers (ED, AM, and MTS) between June to October 2017, and the average duration was 1 hour. Interviews included a review of factors associated with burnout at the organization based on findings from the survey ${ }^{27}$ along with drivers of burnout described in the literature. ${ }^{21,27}$ Participants were asked to identify the most salient factors contributing to burnout using a card sorting exercise, ${ }^{28}$ and were then asked questions about these identified factors (see interview guide in online Appendix 1).

Recruitment continued in tandem with analysis until we reached thematic saturation. ${ }^{29}$ Interviews were transcribed and imported into Atlas.ti software for analysis. The coding team (ED, AM, VM) met weekly to develop a codebook and coding structure. Initial deductive codes were identified from prior research. $^{21,27}$ The coders began with descriptive and open coding using both pre-identified and emergent codes, including proposed strategies for reducing burnout. The second cycle of coding categorized codes into themes using a pattern coding approach, ${ }^{27,30}$ and matched factors to solutions suggested to improve well-being.

\section{RESULTS}

Seventeen of 21 key informants emailed agreed to participate in an interview. Participant characteristics are reported in Table 1. Participants identified the following organizational factors as primary contributors to burnout and identified relevant solutions: (1) workload and work-life balance; (2) organizational structure and culture; (3) support staff, physician autonomy, and leadership; and (4) social support, feeling valued, and meaning in work (see online Appendix 2 for details of all themes and their frequency). Participants also described how physicians currently cope with burnout. While 9 participants agreed that personal practices are associated with burnout, e.g., lack of exercise and sleep, all 17 noted that organizational factors were more important contributors to
Table 1 Characteristics of Interview Participants

\begin{tabular}{ll}
\hline \hline Total interview participants & $\mathbf{1 7}$ \\
\hline Physicians & 15 \\
Primary care & 12 \\
Other sub-specialties & 3 \\
Non-physician system leaders & 2 \\
Female & 11 \\
Male & 6 \\
Physician medical group leaders & 9 \\
Frontline physician (no leadership role) & 6 \\
Average \# of years at organization & 14.6 \\
\hline
\end{tabular}

Note: Leadership roles included leaders of individual departments, specialty groups, and medical group executive leadership

burnout. One participant said, "It does not matter how resilient or positive you are, the work environment, especially in primary care will eventually be a problem."

Participants believed burnout was most acute in adult primary care departments, i.e., Internal Medicine and Family Medicine. They cited several reasons: (1) less revenue and fewer resources for staffing; (2) a higher burden of EHR inbasket messages; (3) responsibility for care coordination, e.g., "everything runs downhill" to primary care; and (4) patients with chronic problems: "They tend to have a lot of needs." Table 2 provides an overall summary of identified contributors to burnout and potential solutions. We describe them below.

\section{Workload and Poor Work-Life Balance: Desktop Medicine}

Many physicians $(n=13)$ reported a significant increase in workload in recent years largely attributable to the EHR and two "pain points": in-basket messages and progress notes. Inbasket messages were described as a "nightmare" and a "fire hose" that never turned off, particularly in primary care. In-basket messages came from patients, colleagues, laboratories, pharmacies, physicians themselves, and the EHR system. ${ }^{27}$ The pressure to complete progress notes and close charts also contributed to what one participant described as the "pajama time problem"31: "You close your charts. You do your in-basket... you're in pajamas, and you're finishing your work for the day."

The solutions suggested to address desktop medicine and work-life balance included reducing and compensating desktop medicine work by "redefining the FTE," enhancing care team support to triage or answer in-basket messages and scribe support to assist with other EHR work such as documentation, along with increasing physicians' flexibility and control over their schedule.

\section{Organizational Structure and Culture: the Push for Productivity}

The reliance on face-to-face visits for revenue was described as problematic due to the growth of uncompensated desktop medicine work. Participants described an organizational culture increasingly focused on growth, productivity, and physician performance metrics, and noted increased discussion of "business" in physician meetings in recent years. 
Table 2 Causes of Burnout and Potential Solutions as Discussed in Interviews

\begin{tabular}{l}
\hline Causes of burnout \\
Increasing desktop medicine time: \\
"I was signing notes until $10 \mathrm{o}$ 'clock at night and then all \\
day on my day off...." \\
"You're losing the revenue we can make for the \\
organization by derailing us doing these [in-basket] \\
messages. There are not enough hours in the day."
\end{tabular}

Decreased work-life balance:

"When you start eating away at your ability to enjoy your family, and you start feeling guilt, it just all falls apart. As women, we have to really support and advocate for one another that your family is really important, and it's more important than work."

Fee-for-service business model

"We get a lot of in-basket messages [because] patients don't want to come in. It's not convenient. It costs money. So, being able to provide that in other ways that's compensated or where it's capitated."

Culture focused on productivity and growth

"We started to take a lot of metrics on everything. That is a little bit stressful to always see that you're in the red..."

"Our panels are unsustainably big... We can't even talk about growth until we stabilize."

Sufficient and stable support staff

"[the health system] will say, 'You need to make more RVUs if you want to pay for that staff,' it's this vicious cycle. I can't make more RVUs if you don't give me the staff to support me, but then I can't get the staff member unless I make the RVUs."

Causes of burnout

Decreasing physician autonomy

"Loss of control, top-down decisions. You may have heard these buzzwords. These are the examples of things that have come along that have stripped away at a physician's sense of autonomy, control, and then therefore leads to responses of feeling burned out."

Leadership roles contribute to burnout

"A lot of what you do [as a leader] is bring bad news to people, like 'Now we don't have the RNs in the evening.' ... that might lead to my burnout because who wants to be spreading bad news to people all the time? I mean, I know there's good news, but a lot of things unfortunately are 'Do more, do more on the hamster wheel."

Decreased collegiality at work

"I have a lot of colleagues that are really burned out. They're just like, 'I come and do my work, and I go home. I can't engage any more than that.' They're just fried."

\section{Feeling de-valued}

"I haven't had somebody say something that I felt highly valued for in a long time. I've been told I'm not seeing enough patients, I'm not doing this, I'm not doing this, I need to do this..."

Loss of meaning in work

"When you say meaning in work, what do you mean? What are things that you're not able to do right now that you want to do more of? Now, we ask [physicians] what they want to do less: 'in basket'. Why not ask them what they want to do more?"

\section{Suggested solutions}

Provide support and/or compensation for this work

1. Compensating desktop medicine work

2. Scheduling desktop medicine time

3. Reducing flow of in-basket messages

4. Expanding the team who can answer/triage messages

5. Scribes

"I'll take care of the half of the emails that really matter. The other half are taken care of by my team."

"Being mindful of what we're sending to the primary care doctor because one more message times 100 is 100 messages."

Reduce workload

1. "Right-size" primary care panels and enforce panel size limits

"You're allowed to go part-time, and that's how people manage... So we have the flexibility of schedule being part-time, which is part of why we hire over [another local health system]."

Evolve the business model

1. Negotiate new agreements with insurers to include payments for virtual care

2. Adopt value-based payment

3. Move from RVU to salary model

4. Allocation of revenue from other sources to primary care

"We've got to be willing to slay the fee-for-service beast a little bit"

Focus on well-being of existing patients and physicians

1. Stabilize the workforce before attempting growth

2. Embracing positive stories and emphasize medicine over business/metrics

"I would say that it would be okay for our organization to go in the red, to invest heavily to have a better tomorrow... and if you want to be successful, you're going to have to take that risk. Otherwise, we're going to continue to have a slow, dwindling death."

Enhance support staff workforce and retention

1. Provide additional support staff

2. Re-allocate existing staff

3. Allocate support staff for all clinic work hours

4. Improve staff retention

"I don't even think we need to add more support staff. I think if we cross-trained and spread some of our licensing out, we could really optimize primary care at the same time we're using people more under their full scope of practice."

Suggested solutions

Maintain and increase physician control \& flexibility

1. Ensure flexible schedules

2. Give departments control over key decisions

3 . Allow telemedicine /virtual visits

"They can tell us how many [appointment] slots they want, and we should be

able to figure out how to staff it... Let us solve that in the department."

Increase leadership support

1. More administrative support

2. Increase allocated FTE time/stipends

3. Provide more training and mentorship

"I loved leadership, but it was a second or third job. And the support for leaders

is not what it could be in terms of just resourcing support and stipends."

Fostering relationships between physicians

1. Provide opportunities and funding for social interactions

2. Promote attendance at physician meetings

3. Embrace fun and positivity and change the culture

"Having more time for friendship, with your colleagues, and it's not about a case..."

Ensure physicians feel valued and heard

1. Reduce the emphasis on metrics

3. Embrace and celebrate accomplishments

4. Identify and respond to physician concerns

"One of the things about feeling highly valued is my opinion counts..."

Restoring meaning in work

1. Restoring focus on patients

2. Physicians work at top of license

"I get to focus on my patients and that's a huge job satisfier for me."

"But meaning in work means I do what a doctor is uniquely qualified to do. And I interact with my patients in a meaningful way.... You'd have to almost say, define the physician role. What are they uniquely qualified to do? Make sure that the workload and job demands fit a physician role."
Physicians noted the limitations of metrics, e.g., a physician whose office visits are all 15 min may appear "efficient," but may not be spending enough time with some patients. Physicians also disliked the variation reduction practice of comparing physicians, 
labeling someone "in the red," and distributing department metrics with physician identities revealed.

Participants suggested that the business model should evolve by changing how revenue is generated and resources are allocated. They suggested that the culture should focus on the well-being of existing patients and physicians. In response to "more and more business-like" physician meetings, they suggested limiting time spent on "business" and instead emphasizing positive stories and discussion of clinical challenges.

\section{Support Staff, Physician Autonomy, and Leadership}

Availability and retention of support staff strongly influenced physician well-being. Primary care physicians noted that medical assistants were only allocated during scheduled patient visit time, so physicians lacked support while working on desktop medicine tasks.

Organizational centralization and standardization efforts reduced physician autonomy and contributed to feelings of disempowerment and burnout. One physician described how the transition to a centralized call center meant that individual physicians' preferences for scheduling were no longer honored, "[we] lost a bunch of workflows that were working pretty well."

Physicians occupying leadership roles noted having minimal or no administrative support, not enough compensated time for leadership work, and insufficient training and mentorship. One former leader described the transition to leadership: "There wasn't a lot of support... 'Now you're a leader. Figure it out...' And that was very alienating for me." Physicians remarked that burnout among leadership had a trickledown effect and led to loss of institutional knowledge and momentum when leadership changed.

Strategies suggested to improve staffing included enhancing care teams so physicians work at the top of their skill level, allocating more support staff to primary care, hiring advanced practice clinicians (APCs), and improving staff retention. Physicians wanted staff to be allocated to them during all the hours they were at the clinic instead of only when face-to-face visits are scheduled. Strategies suggested to enhance physician autonomy included increasing physician control over schedules, and allowing telemedicine or virtual visits. Increasing leadership support, e.g., by increasing administrative support, would save physician leaders time and energy for work which required their skills. They also desired additional training, mentorship, and stipends for leadership work.

\section{Social Support, Feeling Valued, and Meaning in Work}

Physicians described their feelings about work being influenced by three factors (1) social support and camaraderie with colleagues, (2) feeling valued and heard, and (3) finding meaning in work.
Participants noted that a sense of community and social support at work facilitated well-being and was decreasing: "People on the EMR [Electronic Medical Records]... they don't go to the cafeteria. They eat at their desk looking at the computer." Nevertheless, participants told us that their colleagues were a strong positive force: "You want colleagues that are fun to be with, smart, and we have that here. So that's a lot of what keeps people here."

Physicians described an absence of recognition of the positive aspects of their work that led them to feel de-valued, and that being valued required more than a pat on the back.

"If you said, 'We value you, we value your opinion, we know that you're stressed because you get $\mathrm{X}$ amount of messages every day, we're doing this to try to help you.' That to me means that you're highly valued."

Participants remarked that helping patients makes work meaningful, and that burnout was increased by tasks that reduced their face-to-face time with patients, such as desktop medicine time.

Strategies suggested to ensure physicians felt valued and appreciated included reducing the emphasis on productivity, and consciously embracing and celebrating clinical and organizational accomplishments. Participants suggested fostering quality social interactions between colleagues to improve well-being. They pointed to increased organizational efforts to have group lunches, and ad hoc efforts to increase department social events. Physicians also suggested a need to systematically identify and respond to concerns through regular well-being surveys, mentoring programs, and ensuring that physicians' voices are heard, questions are answered, problems are addressed, and meaningful work is prioritized.

\section{Coping with Burnout: Fractional Quitting and Quitting}

Physicians described several coping strategies used to reduce burnout, specifically (1) reducing clinical work hours, dubbed "fractional quitting," (2) leaving leadership roles, (3) switching departments, or (4) leaving the job (for another position elsewhere or to retire). Physicians described coping with long work hours, long commutes, and evening and weekend work by reducing clinical work hours in order to spend more time with family or pursue other interests. Participants reported that in primary care, the majority of physicians worked less than 1.0 FTE and many new hires requested to reduce clinical work hours once they were past the organization's initial 2-year introductory period: "The first thing people ask for is to cut back their hours."

One physician leader described a pattern in primary care she called the "vicious cycle":

"I'm burnt out at 1.0 now I'm going to cut back to .75 time. Now I'm making fewer RVUs, so they'll cut my staff, but I'm working the same numbers of hours. Once I get to 0.5 and am still working a full time job 
effectively, 35 hours, then what is my only option? I'll work for [local biotech company] or be a same day doc where I don't have to work on in-basket."

Another physician, a self-described "recovering leader," relinquished a leadership role to re-allocate time to patient care and improve personal well-being. Participants noted that female physicians were more likely to reduce FTE and prioritize family over work.

"We know what's actually happening is people fractionally quit, so that they can manage the in-basket. And it's not just women anymore, but so often it lands on some of the female providers because they are managing children's schedules. And I honestly think women are like, you know what? I'm working parttime because it's how I can cope."

\section{DISCUSSION}

Many of the factors causing, and strategies to improve, burnout, mentioned by participants, are greatly intertwined. Participants identified organizational factors as having the biggest impact on burnout and well-being, including the fee-for-service business model, the growth of EHR-related tasks, and growing focus on productivity metrics. These factors contributed to increasing workloads, loss of autonomy, and feeling de-valued. The exacerbation of stressors in primary care, e.g., lack of support staff, increased in-basket messages, and a shortage of physicians, led to a feeling that primary care is facing a crisis. Leaders also expressed a need for enhanced support, and research suggests that helping leaders succeed could have a powerful positive effect on the whole care team. ${ }^{32,33}$

Strategies suggested to improve well-being included: decreasing and/or compensating desktop medicine work; $;{ }^{27}$ ensuring adequate support staff; ${ }^{34}$ enhancing physician autonomy; and ensuring physicians feel valued and heard. ${ }^{21}$ Participants also had recommendations that had not previously been discussed in the literature, including providing support staff for all physician clinic work hours (not just patient visit hours), allocating or scheduling time for responding to increasing inbasket messages ${ }^{27}$ and ensuring leadership affirms the value of frontline physicians by telling them, and more importantly, demonstrating through action that "your opinion counts." Individual factors such as sleep, exercise, and mindfulness were cited as helpful, but insufficient to counter the potent organizational forces contributing to burnout.

The feasibility of implementing the solutions suggested by physicians merits further attention. Some have the potential to be implemented relatively quickly, e.g., reducing the emphasis on metrics, enforcing panel size limits, celebrating accomplishments, and spending more time on medicine at physician meetings, while others would require substantial lead time and potentially challenging negotiations with organizational stakeholders, e.g., increased number or reallocation of support staff, re-allocating how revenue is distributed throughout the organization, ensuring physicians feel valued and heard, and negotiations with internal and external stakeholders to change reimbursement and compensation models.

These interviews highlight how physician well-being is linked to staffing and the EHR. Physicians who report their clinics are under-staffed or have high staff turnover exhibit higher rates of burnout. ${ }^{34}$ Reducing desktop medicine and increasing staffing were the most commonly mentioned strategies for improving well-being. Expanded care teams have demonstrated value in reducing physician EHR time in primary care. ${ }^{20}$ Assisting physicians with certain clinical tasks may also increase physician well-being. ${ }^{6}$ This organization has taken recent steps to improve well-being, including creating an office of physician wellness, piloting multidisciplinary in-basket support, providing enhanced leadership development support and training, and implementing a primary care transformation program including in-basket support from APCs. Physicians also stressed that relationships with colleagues and patients enhanced their own well-being. In addition, increasing physician well-being will likely require addressing the well-being of all clinic staff.

Changes in the profession of medicine and the demographics of the workforce may also be escalating physician burnout. In recent decades, health systems have grown and turned their attention to standardization and productivity metrics. Ariely and Lanier ${ }^{35}$ suggest that physician work environments are becoming a "fixing-people production line." Some evidence suggests that organization size is related to burnout; physicians in small solo practices have much lower burnout rates than physicians in large groups, perhaps because of greater autonomy. ${ }^{36}$ These trends are accompanied by an increasingly female workforce who typically bear greater responsibility for caring for young children, often choose to reduce work hours rather than reduce family time, ${ }^{37-39}$ and may face additional discrimination. ${ }^{40}$

These interviews contribute personal insights about how physicians experience burnout and solutions they believe would enhance well-being, which are often not available in structured surveys. ${ }^{41}$ It is essential for organizations to learn from their own physicians when developing strategies to improve well-being. Well-intentioned efforts promoting individual-level interventions (e.g., mindfulness training) to improve workplace wellness may be alienating if they are at odds with physician perceptions of organizational pressures as the root cause of burnout.

\section{Limitations}

Interview participants worked at one predominately fee-forservice organization with an oversampling of physicians in leadership roles and primary care. Future research should seek the perspectives of physicians in other specialties with high rates of burnout. ${ }^{7,42}$ This organization's location in northern California with its strong economy, growing population, and 
high cost of living may pose additional challenges for physicians and staff. This organization was an early adopter of the EHR and patient portals, and its physicians may receive an above average number of in-basket messages. However, other systems may soon face similar increases in in-basket work.

\section{Conclusion}

Participants in these interviews perceived that the causes of burnout were partly due to the health system's increased focus on productivity in recent years. Ultimately, the biggest gains in physician well-being may require broader changes to how financial incentives are structured in healthcare, changing culture, redistributing EHR work among physicians and support staff, and redesigning current EHR systems and staffing models, so that physicians spend more time doing work they find meaningful.

Acknowledgments: We acknowledge the guidance and support of members of the Palo Alto Foundation Medical Group well-being committee, especially Dr. Ruth Steinberg.

Corresponding Author: Ellis C. Dillon, PhD; Palo Alto Medical Foundation Research Institute, Palo Alto, CA, USA (e-mail:dillone@sutterhealth.org).

Funding information This project was supported by funding from the Palo Alto Foundation Medical Group and Palo Alto Medical Foundation Philanthropy.

Some of the results reported here were also reported in a poster at the 2018 Health Care Systems Research Network (HCSRN) conference, April 12, 2018, Minneapolis.

\section{Compliance with ethical standards:}

Conflict of interest: The authors declare that they do not have a conflict of interest.

\section{REFERENCES}

1. Arndt BG, Beasley JW, Watkinson MD, et al. Tethered to the EHR: Primary care physician workload assessment using EHR event log data and time-motion observations. Ann Fam Med. 2017;15(5):419-426.

2. Tai-Seale $\mathbf{M}$, Olson $\mathbf{C W}, \mathbf{L i} \mathbf{J}$, et al. Electronic health record logs indicate that physicians split time evenly between seeing patients and desktop medicine. Health Aff (Millwood). 2017;36(4):655-662.

3. Sinsky CA, Dyrbye LN, West CP, Satele D, Tutty M, Shanafelt TD. Professional satisfaction and the career plans of US physicians. Mayo Clin Proc. 2017;92(11):1625-1635.

4. Shanafelt TD, Hasan O, Dyrbye LN, et al. Changes in burnout and satisfaction with work-life balance in physicians and the general US working population between 2011 and 2014. Mayo Clin Proc. 2015;90(12):1600-1613.

5. Rabatin J, Williams E, Baier Manwell L, Schwartz MD, Brown RL, Linzer M. Predictors and outcomes of burnout in primary care physicians. J Prim Care Community Health. 2016;7(1):41-43.

6. Kim LY, Rose DE, Soban LM, et al. Primary care tasks associated with provider burnout: Findings from a veterans health administration survey. J Gen Intern Med. 2018;33(1):50-56.

7. Shanafelt T, Boone $\mathbf{S}$, Tan $\mathbf{L}$, et al. Burnout and satisfaction with worklife balance among US physicians relative to the general US population. Arch Intern Med. 2012;172(18): 1377-1385.
8. Shanafelt TD, Balch CM, Dyrbye L, et al. Special report: suicidal ideation among American surgeons. Arch Surg. 2011;146(1):54-62.

9. Oreskovich MR, Shanafelt T, Dyrbye LN, et al. The prevalence of substance use disorders in American physicians. Am J Addict. 2015;24(1):30-38.

10. Linzer M, Manwell L, Williams $\mathbf{E}$, et al. Working conditions in primary care: physician reactions and care quality. Ann Intern Med. 2009;151(1):28.

11. Henriksen K, Battles JB, Marks ES, et al. Organizational climate, stress, and error in primary care: the MEMO study. Rockville, MD: Agency for Healthcare Research and Quality;2005.

12. Halbesleben JR, Rathert C. Linking physician burnout and patient outcomes: exploring the dyadic relationship between physicians and patients. Health Care Manag Rev. 2008;33(1):29-39.

13. Hall LH, Johnson J, Heyhoe J, Watt I, Anderson K, O'connor DB. Exploring the impact of primary care physician burnout and well-being on patient care: a focus group study. J Patient Saf. 2018.

14. Shanafelt TD, Bradley KA, Wipf JE, Back AL. Burnout and selfreported patient care in an internal medicine residency program. Ann Intern Med. 2002;136(5):358-367.

15. Haas JS, Cook EF, Puopolo AL, Burstin HR, Cleary PD, Brennan TA. Is the professional satisfaction of general internists associated with patient satisfaction? J Gen Intern Med. 2000;15(2):122-128.

16. Lafreniere JP, Rios R, Packer H, Ghazarian S, Wright SM, Levine RB. Burned out at the bedside: patient perceptions of physician burnout in an internal medicine resident continuity clinic. J Gen Intern Med. 2016;31(2):203-208.

17. Bodenheimer T, Sinsky C. From triple to quadruple aim: care of the patient requires care of the provider. Ann Fam Med. 2014;12(6):573-576.

18. Spinelli WM. The phantom limb of the triple aim. Mayo Clin Proc. 2013;88(12):1356-1357.

19. Panagioti M, Panagopoulou E, Bower P, et al. Controlled interventions to reduce burnout in physicians: A systematic review and meta-analysis. JAMA Intern Med. 2017;177(2): 195-205.

20. Pozdnyakova A, Laiteerapong $\mathbf{N}$, Volerman A, et al. Impact of medical scribes on physician and patient satisfaction in primary care. J Gen Intern Med. 2018:1-7.

21. Shanafelt TD, Noseworthy JH. Executive leadership and physician wellbeing: nine organizational strategies to promote engagement and reduce burnout. Mayo Clin Proc. 2017;92(1):129-146.

22. Goldhagen BE, Kingsolver $\mathbf{K}$, Stinnett SS, Rosdahl JA. Stress and burnout in residents: impact of mindfulness-based resilience training. Adv Med Educ Pract. 2015;6:525-532.

23. Rosdahl JA, Kingsolver K. Mindfulness training to increase resilience and decrease stress and burnout in ophthalmology residents: a pilot study. Invest Ophthalmol Vis Sci. 2014;55(13):5579-5579.

24. Zwack J, Schweitzer J. If every fifth physician is affected by burnout, what about the other four? Resilience strategies of experienced physicians. Acad Med. 2013;88(3):382-389.

25. Picard J, Catu-Pinault A, Boujut E, Botella M, Jaury P, Zenasni F. Burnout, empathy and their relationships: a qualitative study with residents in General Medicine. Psychol Health Med. 2016;21(3):354-361.

26. Stevenson AD, Phillips CB, Anderson KJ. Resilience among doctors who work in challenging areas: a qualitative study. $\mathrm{Br} \mathrm{J}$ Gen Pract. 2011;61(588):e404-e410.

27. Tai-Seale M, Dillon EC, Yang Y, et al Physicians' Well-Being Linked To InBasket Messages Generated By Algorithms In Electronic Health Records. Health Aff. 2019;38(7):1073-1078.

28. Cataldo EF, Johnson RM, Kellstedt LA, Milbrath LW. Card sorting as a technique for survey interviewing. Public Opin Q. 1970;34(2):202-215.

29. Guest G, Bunce A, Johnson L. How many interviews are enough? An experiment with data saturation and variability. Field Methods. 2006; 18(1):59-82.

30. Saldaña J. The coding manual for qualitative researchers. 3E Third edition . ed. Los Angeles; London: SAGE; 2016.

31. Berg S. Family doctors spend 86 minutes of "pajama time" with EHRs nightly. American Medical Association 2017; https://www.ama-assn. org/practice-management/digital/family-doctors-spend-86-minutes-pajama-time-ehrs-nightly. Accessed January 17, 2019.

32. Shanafelt TD, Gorringe G, Menaker R, et al. Impact of organizational leadership on physician burnout and satisfaction. Mayo Clin Proc. 2015;90(4):432-440. 
33. Williams ES, Manwell LB, Konrad TR, Linzer M. The relationship of organizational culture, stress, satisfaction, and burnout with physicianreported error and suboptimal patient care: Results from the MEMO study. Health Care Manag Rev.. 2007;32(3):203-212 https://doi.org/10. 1097/1001.HMR.0000281626.0000228363.0000281659.

34. Helfrich CD, Simonetti JA, Clinton WL, et al. The association of teamspecific workload and staffing with odds of burnout among VA primary care team members. J Gen Intern Med. 2017;32(7):760-766.

35. Ariely D, Lanier WL. Disturbing trends in physician burnout and satisfaction with work-life balance. Paper presented at: Mayo Clinic Proceedings. 2015

36. Blechter B, Jiang N, Cleland C, Berry C, Ogedegbe $\mathbf{O}$, Shelley $\mathbf{D}$. Correlates of burnout in small independent primary care practices in an urban setting. J Am Board Fam Med. 2018;31(4):529-536.

37. Hedden L, Barer ML, Cardiff K, McGrail KM, Law MR, Bourgeault IL. The implications of the feminization of the primary care physician workforce on service supply: a systematic review. Hum Resour Health. 2014;12:32.

38. Barnett RC, Gareis KC, Carr PL. Career satisfaction and retention of a sample of women physicians who work reduced hours. J Women's Health (Larchmt). 2005; 14(2):146-153.

39. Barnett RC, Gareis KC. Full-time and reduced-hours work schedules and marital quality a study of female physicians with young children. Work Occup. 2002;29(3):364-379.

40. Halley MC, Rustagi AS, Torres JS, et al. Physician mothers' experience of workplace discrimination: a qualitative analysis. BMJ. 2018;363:k4926.

41. Geertz C. Thick Description: Toward an Interpretive Theory of Culture. The Interpretation of Cultures: Selected Essays. New York: Basic Books; 1973:3-30.

42. Arora M, Asha S, Chinnappa J, Diwan AD. Review article: burnout in emergency medicine physicians. Emerg Med Australas. 2013;25(6):491495 .

Publisher's Note Springer Nature remains neutral with regard to jurisdictional claims in published maps and institutional affiliations. 